\title{
Novel Cocrystals of Glipizide: Green Supramolecular Mechanosynthesis
}

\author{
Dimpy Rani ${ }^{1}$, Sarb Sukhmani Kaur Dargan², Parnika Goyal ${ }^{2}$ and Renu Chadha ${ }^{2 *}$ \\ ${ }^{1}$ G D Goenka University, Gurgaon-122103, India \\ ${ }^{2}$ University Institute of Pharmaceutical Sciences, Panjab University, Chandigarh-160014, India
}

*Corresponding author: Renu Chadha, Department of Pharmaceutical Sciences, University Institute of Pharmaceutical Sciences, Panjab University, Chandigarh-160014, India.

Received Date: October 26, 2018

Published Date: November 13, 2018

\begin{abstract}
The present research demonstrates the five novel cocrystals of glipizide (GPZ) with picolinic acid (PA), adipic acid (AA), isonicotinic acid (INA), fumaric acid (FA) and sorbic acid (SRA). The cocrystals were prepared via green supramolecular mechanosynthesis approach and exhibited 1:1 stoichiometric ratio. The cocrystals were characterized by thermal (DSC) and spectroscopic (PXRD, FTIR and SSNMR) techniques. PXRD data was used to elucidate the crystal structure. GPZ-PA, GPZ-AA, GPZ-INA, GPZ-FA and GPZ-SRA were found to be crystallized in triclinic and monoclinic crystal systems, which exist in P-1, P1 and P21 space groups. Remarkable improvement in solubility and intrinsic dissolution rate (IDR) was observed along with significant reduction in rat blood serum glucose level and high $\mathrm{C}_{\max }$ in comparison to GPZ.
\end{abstract}

Keywords: Glipizide; Cocrystals; Crystal structure; Solubility; Pharmacological studies

Abbreviations: GPZ: Glipizide; PA: Picolinic Acid; AA: Adipic Acid; INA: Isonicotinic Acid; FA: Fumaric Acid; SRA: Sorbic Acid; API: Active Pharmaceutical Ingredients; GRAS: Generally Regarded as Safe; LAG: Liquid Assisted Grinding; DSC: Differential Scanning Calorimeter; PXRD: Powder X-ray Diffraction; FTIR: Fourier Transform Infrared; SSNMR: Solid State Nuclear Magnetic Rasonance

\section{Introduction}

Drug development via the traditional synthetic methods involves the usage of a large quantity of chemicals which in turn generates by-products that adversely affect the environment [1]. In this case, it is the need of the hour to probe the environmentally friendly i.e., green alternative synthetic strategies, which are more selective and economical for the development and optimization of the existing APIs (Active Pharmaceutical Ingredients) at the industrial scale [2-4]. As compared to the solvent based chemical reactions, the solid-state reactions are the viable green approach to achieve these goals in an efficient manner. Solid state mechanochemistry is of great interest as it caters the opportunity to widen the scope of synthetic reactions and build up more sustainable methods. The other alluring aspects of these ecofriendly reactions include little or no use of solvents, high yield and purity of products, stochiometric and polymorphic control, cost effectiveness and time efficiency [3,5-7]. Mechanosynthesis has emerged as a versatile tool for the supramolecular synthesis in the pharmaceutical field. Modification in the intermolecular interactions leads to the variation in the crystal lattice, which can result in the improvement of physicochemical properties, and in turn enhances the performances of pharmaceutical solids [8]. Based on these concepts, the manuscript has been emphasized to tailor supramolecular architecture by incorporating counter molecules in the lattice of API, leading to cocrystals. The use of GRAS (Generally Regarded as Safe) conformers, and acceptable solvents in nominal amount makes pharmaceutical cocrystallization technique as a part of green process. Out of various methods, liquid-assisted grinding (LAG) is preferable and commonly used method for the preparation of pharmaceutical cocrystals. The catalytic amount of solvent in the reactions enhances the molecular mobility and accelerates the reaction rate. Moreover, the addition of solvent also leads to generation of more crystalline products with control over polymorphic behavior [9-14]. The legal benefits and consideration as a new chemical entity of cocrystals also aids in making it a preferable choice to explore.

The present work is focused on the synthesis, characterization and evaluation of the cocrystals of glipizide (GPZ; Figure 1), an oral short-acting hypoglyceamic agent. GPZ is prescribed widely for treating type II diabetes and thus, grabs the attention to improve the clinical performance by ameliorating the setback of dissolution 
limited solubility. There are diverse approaches that have been adopted to ameliorate the solubility of GPZ. These include solid dispersion, hydrotropy, micellar solubilization, nanoparticles, cyclodextrin complexes and co-inclusion complex formation [15-18]. The availability of numerous hydrogen bond donor and acceptor in GPZ and the ability of the cocrystals to customize the physicochemical properties at root level without forming or breaking the covalent bonds make the cocrystals a viable mean to enhance the solubility and the clinical performance of GPZ.

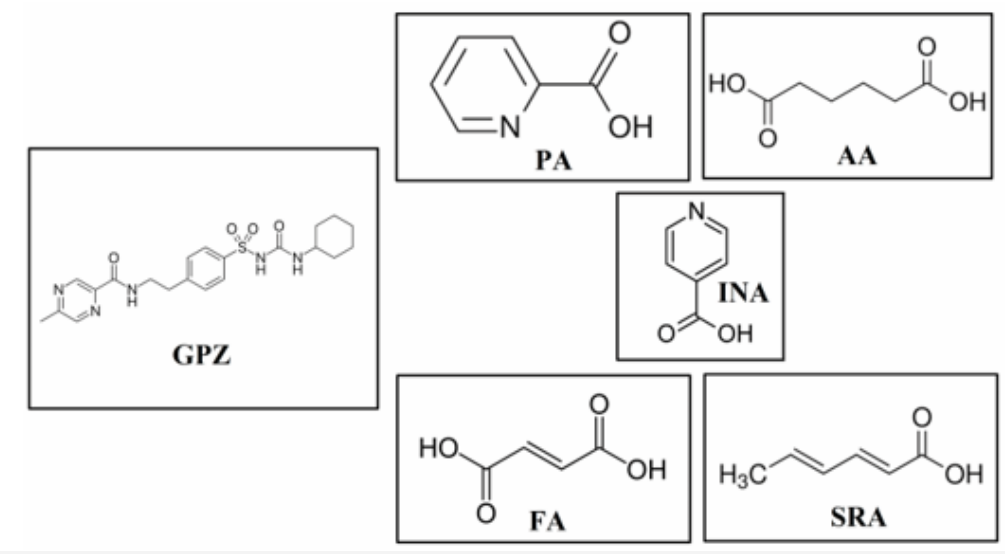

Figure 1: Chemical structure of GPZ and coformers.

As far as we are aware, not much work has been done on the cocrystallization of glipizide except one recent report in which authors have given four cocrystals of glipizide. However, they have not emphasized on their characterization as well as the structural parameters to identify hydrogen bonding in the crystal lattice which is vital for the stabilization of the cocrystals. In this manuscript, the isolation of pharmaceutically relevant cocrystals of glipizide (GRAS coformers: picolinic acid (PA), adipic acid (AA), isonicotinic acid (INA), fumaric acid (FA) and sorbic acid (SRA)) is described with focus on their proper characterization and structure elucidation (Figure 1).

\section{Experimental}

\section{Materials}

GPZ ( $\geq 99 \%$ ) was obtained from La Pharma Pvt Ltd, Ludhiana, India, as a gift sample. All the chemicals and solvents were purchased from Sigma-Aldrich or E. Merck Ltd, and used as received.

\section{Mechanosynthesis of cocrystal}

Liquid assisted (solvent: ethanol) solid state grinding of the binary mixtures of GPZ with selected cocrystal formers (GPZ-PA (1:1; 44.55mg:12.31mg), GPZ-AA (1:1; 45.26mg:12.31mg), GPZINA (1:1; 45.26mg:12.31mg), GPZ-FA (1:1; 45.26mg:11.60mg), and GPZ-SRA (1:1; 45.26mg:11.21mg)) were performed. GPZ and different coformers, in their respective molar ratio were ground for 60 minutes in a mortar and pestle, with drop by drop addition of solvent simultaneously.

\section{Differential Scanning Calorimetry (DSC)}

DSC scans were recorded on Q 20 (TA-Instruments Inc., USA) with a refrigerated cooling system, at a heating rate of $10^{\circ} \mathrm{C} / \mathrm{min}$ under dry nitrogen purge (flow rate $50 \mathrm{ml} / \mathrm{min}$ ). The samples were packed in aluminium pans and run for scan in the temperature range of $25-250^{\circ} \mathrm{C}$, using blank aluminium pan as reference.

\section{Powder X-ray Diffraction (PXRD)}

For the qualitative analysis of the cocrystals, PANalytical X'Pert Pro X-ray powder diffractometer (The Netherlands, Holland) with $\mathrm{Cu}$ source, at voltage of $40 \mathrm{kV}$ and current $40 \mathrm{~mA}$ was used. The samples were scanned in the range of $5^{\circ}-45^{\circ}(2 \theta)$ with step size $0.02^{\circ} \theta / \mathrm{min}$, scan rate $3^{\circ} / \mathrm{min}$ and high resolution.

\section{Fourier Transform Infrared (FTIR) spectroscopy}

The samples were dispersed in potassium bromide and the pellet was prepared. The spectra of the pellets were collected using spectrum two IR spectrometer (Perkin Elmer, England). Each spectrum was derived from 4 accumulative scans in the range of $400-4000 \mathrm{~cm}^{-1}$ at a spectral resolution of $4 \mathrm{~cm}^{-1}$.

\section{Crystal structure determination from PXRD}

The determination of the crystal structures of all the cocrystals was done by Material studio (BIOVIA 7.0) software using PXRD. The PXRD were obtained from PANalytical X'Pert Pro X-ray powder diffractometer (The Netherlands, Holland). The PXRD patterns were obtained in the range of $5^{\circ}-45^{\circ}(2 \theta)$ with step size $0.02^{\circ} \theta$ / min, scan rate $3^{\circ} / \mathrm{min}$ and with 3250 reflections. These PXRDs were used to determine the crystal structures of the cocrystals. The process of analyzing the cell parameters consisted of four main steps: Indexing along with pawley refinement, structure solution, and reitveld refinement. Indexing is the crucial step in the structure determination, so the PXRD was pretreated by subtracting the background and smoothing followed by stripping. Diffraction peaks were searched in the range of $5^{\circ}-40^{\circ} 2 \theta$ and indexing was performed by X-CELL method, followed by pawley refinement, which generated the unit cell. Powder solve was performed on the unit cell with geometrically optimized GPZ and coformers. The chemical structures were optimized by DMol3 and simulated annealing algorithm in powder solve was used to adjust the position, orientation and the conformation of the structures in unit 
cell. Afterwards, reitveld refinement was done to get best possible crystal structure with least difference between experimental and calculated powder diffraction pattern.

\section{Solid-state NMR (SSNMR) spectroscopy}

Solid-state ${ }^{13} \mathrm{C}$ NMR spectra were obtained from Jeol-ECX 400 $\mathrm{MHz}$ spectrometer, operating at resonant frequency of $100 \mathrm{MHz}$. The data was collected at $273 \mathrm{~K}$ to minimize frictional heating effects. The spectra were referenced to methylene carbon of glycine ( $\delta$ glycine $=43.3 \mathrm{ppm})$ and then chemical shifts were recalculated to TMS. The other acquisition parameters for ${ }^{13} \mathrm{CPMAS}$ were: contact time, $3.5 \mathrm{~ms}$; acquisition time, $29.0 \mathrm{~ms}$; and relaxation delay, $5 \mathrm{~s}$.

\section{Equilibrium solubility studies}

The equilibrium solubility studies were performed by the method reported by Higuchi and conners [19] i.e., shake flask method. Excess amount of GPZ and its cocrystals were agitated for 24 hours at $200 \mathrm{rpm}$ in a vial containing $5 \mathrm{ml}$ of phosphate buffer (pH 7.4) using water bath shaker MSW-275 Macroscientific works, Delhi at $37{ }^{\circ} \mathrm{C}$. The samples were filtered through $0.45 \mu \mathrm{m}$ membrane filter and quantified by HPLC.

\section{Intrinsic dissolution studies (IDR)}

IDR studies were performed using rotating disk dissolution test apparatus, DS 8000 (Lab India Analyticals) in phosphate buffer (pH 7.4) for 4 hours. The powder of GPZ and cocrystals (equivalent to $150 \mathrm{mg} \mathrm{GPZ)} \mathrm{was} \mathrm{compressed} \mathrm{to} \mathrm{a} 0.5 \mathrm{~cm}^{2}$ disk with hydraulic press. The disk was placed in $500 \mathrm{ml}$ phosphate buffer ( $\mathrm{pH} \mathrm{7.4)}$, preheated at $37^{\circ} \mathrm{C}$ and rotated at $150 \mathrm{rpm}$. The samples were withdrawn $(5 \mathrm{ml})$ manually at different intervals of time and the quantity of buffer in jar was maintained by adding fresh buffer equal to withdrawn quantity, filtered through $0.45 \mu \mathrm{m}$ membrane filter and quantified by HPLC.

\section{In Vivo studies}

The pharmacodynamic and pharmacokinetic studies of GPZ and its cocrystals were performed on diabetic rats (male wistar rats; 3-4-week-old; 150-200 g). Streptozotocin with nicotinamide solution ( $45 \mathrm{mg} / \mathrm{kg}$; prepared in citrate buffer $(0.1 \mathrm{M})$ of $\mathrm{pH} 4.4)$ was injected via intraperitoneal route and the rats were diabetic after 48 hours [20]. GPZ and cocrystals equivalent to GPZ dose (5 $\mathrm{mg} / \mathrm{kg}$, suspended in $2 \%$ sodium CMC [21]) administered orally to the rats. The extent of reduction in blood glucose was measured at different intervals of time $(0,0.5,1,2,3,4,5,6$ and $8 \mathrm{hrs})$. An enzymatic GOD - POD (glucose oxidase peroxidase) method [22] was used to analyze the concentration of glucose in blood. For the pharmacokinetic study, the blood samples from diabetic rats were withdrawn at different intervals of time for 8 hours. The plasma samples were quantified by HPLC and the pharmacokinetic parameters were calculated using PKSolver: An Add-in program [23], based on the linear trapezoidal method.

In both studies, the blood samples were withdrawn from retrobulbar venous plexus and the results were expressed in percentage mean \pm SEM. The obtained data were compared statistically with control groups by Student's t test with $\mathrm{p}<0.05$ as the level of significance, using GraphPad Prism 6.0 software.

\section{HPLC method}

HPLC (Waters; Photodiode Array Detector) and Thermofisher $\mathrm{C}_{18}$ column $(4.6 \mathrm{~mm} \times 250 \mathrm{~mm} \times 5 \mu \mathrm{m})$ were used to quantify the GPZ concentration using calibration plot. The samples (injection volume $10 \mu \mathrm{l}$ ) were eluted with isocratic mobile phase containing a mixture of acetonitrile: water (50:50) of $\mathrm{pH} 3$ (pH was adjusted with orthophosphoric acid) in 10 minutes with flow rate $0.8 \mathrm{ml} /$ min. GPZ peak was detected at $227 \mathrm{~nm}$ with retention time $6.6 \mathrm{~min}$.

\section{Results and Discussion}

DSC

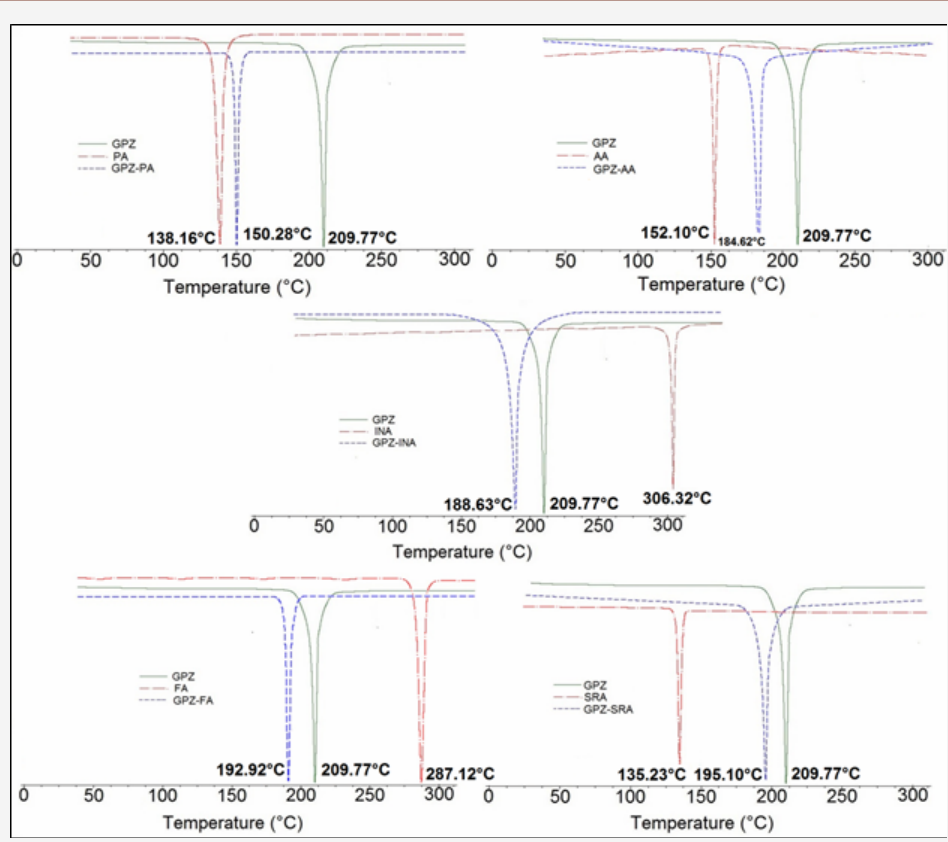

Figure 2: DSC thermograms of GPZ, coformers and cocrystals. 
DSC thermograms (Figure 2) of the prepared solid forms namely GPZ-PA, GPZ-AA, GPZ-INA, GPZ-FA and GPZ-SRA showed a single melting endotherm at $150.28^{\circ} \mathrm{C}, 184.62^{\circ} \mathrm{C}, 188.63^{\circ} \mathrm{C}, 192.92^{\circ} \mathrm{C}$ and $195.10^{\circ} \mathrm{C}$, respectively. These melting peaks are sharp and different from the GPZ $\left(209.77^{\circ} \mathrm{C}\right)$ and respective coformers $\left(\mathrm{PA}-138.16^{\circ} \mathrm{C}\right.$; AA $-152.10^{\circ} \mathrm{C}$; INA- $306.32^{\circ} \mathrm{C} ; \mathrm{FA}-287.12^{\circ} \mathrm{C}$; SRA- $135.23^{\circ} \mathrm{C}$ ). The melting peak of GPZ-PA, GPZ-AA and GPZ-SRA is positioned between the endotherms of GPZ and corresponding coformers, which indicates the likelihood of cocrystal formation. In contrast to these, the endothermic peak of GPZ-INA and GPZ-FA appears before the melting peaks of GPZ and their respective coformers, which suggest the synthesis of either cocrystal or eutectic. However, the
DSC of the physical mixtures (given in supporting data; Figure S1) of all the crystalline phases in stoichiometric ratio 1:1 show the two broad peaks, representative of individual components. This corroborates the formation of cocrystals and at the same time negates the eutectic formation in GPZ-INA and GPZ-FA (Figure 2).

\section{PXRD}

The peaks in the PXRD patterns are the reflections from the atomic planes [24] in the crystal lattice and are the fingerprint for any crystalline material. The PXRD pattern of the obtained solid forms is unique and different from their parent components (Figure 3 ), indicating the generation of a new crystalline phase.

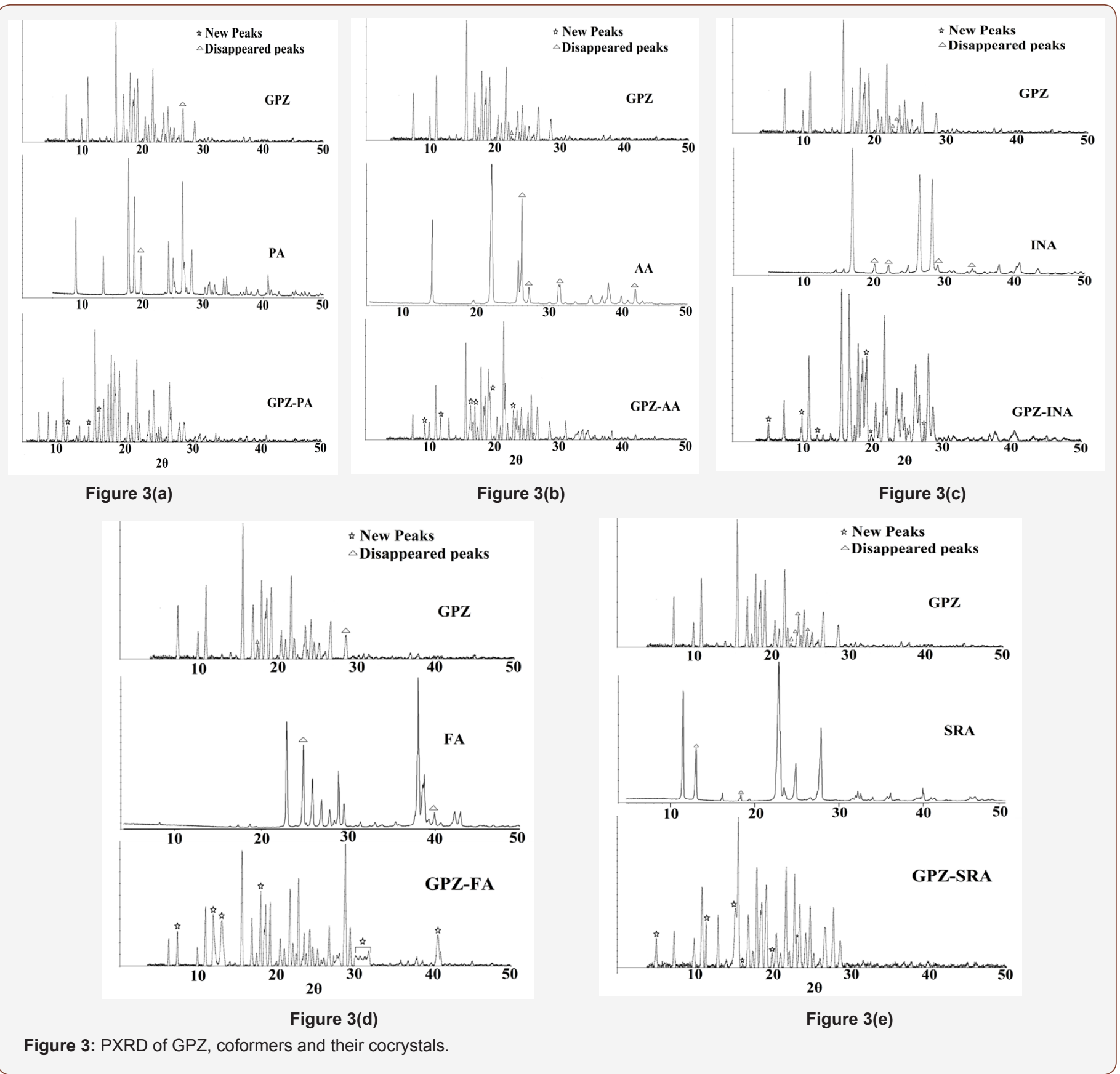

GPZ-PA witness the appearance of many new peaks at $11.49^{\circ}$, $14.81^{\circ}, 16.21^{\circ}$ along with the disappearance of characteristic peaks of GPZ $\left(26.74^{\circ}\right)$ and PA $\left(19.09^{\circ}\right)$. Besides this, few peaks of GPZ at $17.44^{\circ}$ and $24.24^{\circ}$ merged with peaks of PA at $17.61^{\circ}$ and $24.22^{\circ}$ respectively, to give a single and broad peak correspondingly at $17.56^{\circ}$ and $24.20^{\circ}$. Moreover, the peaks of GPZ, positioned at $18.41^{\circ}$ and $18.62^{\circ}$ merged with peak of PA at $18.53^{\circ}$ to give a split peak at $18.49^{\circ}$ and $18.67^{\circ}$. 
In GPZ-AA, new peaks at $10.10^{\circ}, 11.62^{\circ}, 16.20^{\circ}, 16.46^{\circ}, 16.84^{\circ}$, $19.35^{\circ}, 22.80^{\circ}$ and $26.30^{\circ}$ were observed. The peaks at $22.52^{\circ}$ of $\mathrm{GPZ}$, at $26.08^{\circ}, 27.07^{\circ}, 31.52^{\circ}$ and $42.26^{\circ}$ of AA have disappeared. Apart from it, the peak of GPZ at $21.74^{\circ}$ merged with $21.76^{\circ}$, a peak of AA, to give a broad and single peak at $21.75^{\circ}$ in GPZ-AA.

Some new peaks, in GPZ-INA, at $5.03^{\circ}, 10.03^{\circ}, 11.72^{\circ}, 18.83^{\circ}$, $19.83^{\circ}$ and $27.65^{\circ}$ appeared while some characteristic peaks at $22.52^{\circ}$ and $23.27^{\circ}$ of $\mathrm{GPZ}$ and at $20.12^{\circ}, 22.09^{\circ}, 29.06^{\circ}, 34.02^{\circ}$ of INA have disappeared.

In GPZ-FA, a few new peaks at $8.55^{\circ}, 11.97^{\circ}, 12.71^{\circ}, 18.37^{\circ}$, $30.69^{\circ}, 31.20^{\circ}, 31.91^{\circ}, 32.30^{\circ}$ and $41.87^{\circ}$ appeared, accompanied with disappearance of some of the peaks of GPZ at $17.44^{\circ}, 28.60^{\circ}$ and of $\mathrm{FA}$, at $24.80^{\circ}, 39.93^{\circ}$.

In GPZ-SRA, new peaks were observed at $5.32^{\circ}, 11.19^{\circ}, 15.30^{\circ}$, $19.50^{\circ}, 22.96^{\circ}$ while some characteristic peaks at $22.52^{\circ}, 23.27^{\circ}$, $23.51^{\circ}, 23.77^{\circ}, 24.64^{\circ}$ of $\mathrm{GPZ}$ and at $13.00^{\circ}, 18.31^{\circ}$ of SRA have disappeared. A few nearby peaks of GPZ and SRA merged to give either a new peak or a broad peak.

Besides these changes, many characteristics peaks of both GPZ and respective coformers, shifted significantly from their positions. All these noticeable changes in the PXRD are the indicator of the generation of new crystalline phases (Figure 3).

\section{FTIR}

FTIR spectra help to investigate the vibrational changes in the functional groups during supramolecular synthesis. In the cocrystals of GPZ, the significant changes have been seen in the sulfonylurea and amide group of GPZ and carboxylic acid groups of coformers. The changes in the wavenumber of different involved groups have been given in supporting information in Table S1 and FTIR spectra are given in Figure S2. The absence of any new peak in the region of $1650 \mathrm{~cm}^{-1}$ to $1550 \mathrm{~cm}^{-1}$, negates the likelihood of proton transfer during cocrystallization. The variations in the position of characteristic peaks are indicative of some interactions between GPZ and coformers.

\section{Crystal structure determination from PXRD}

The importance of the crystal structure determination can be marked as the physicochemical properties of the compounds are the outcome of the packing of the molecules and the interactions among them. As the crystallization of the ground powder could not yield the suitable crystals for single crystal analysis. So, the crystal structure was determined using the PXRD pattern. The pictures for the crystallized powders are given in (Figure 4).

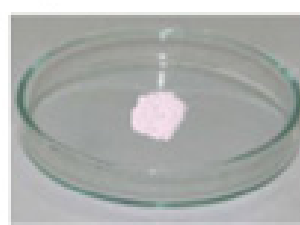

(a)

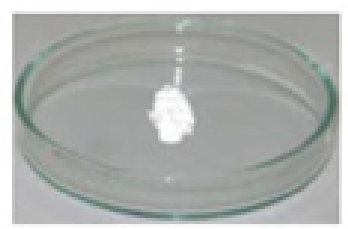

(b)

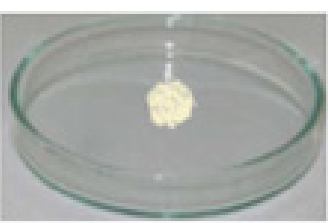

(c)

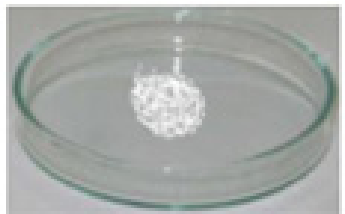

(d)

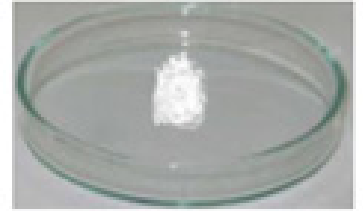

(e)

Figure 4: Pictures of the obtained crystallized cocrystals (a) GPZ-PA (b) GPZ-AA (c) GPZ-INA (d) GPZ-FA (e) GPZ-SRA.

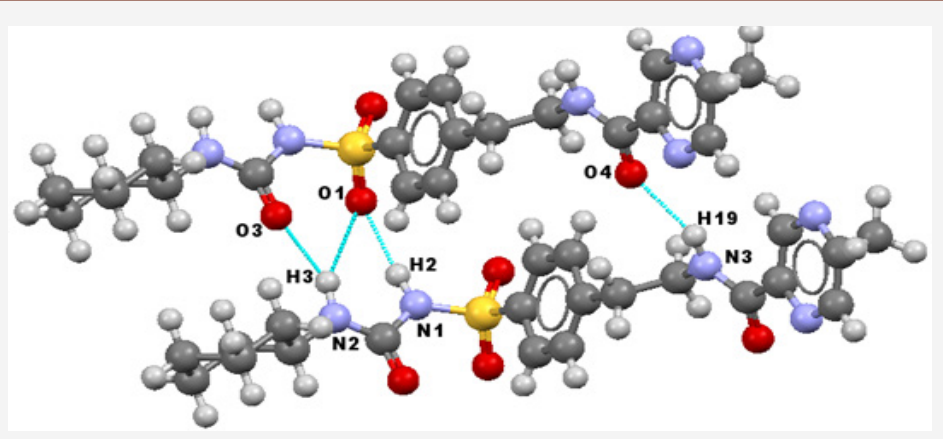

Figure 5 (a)

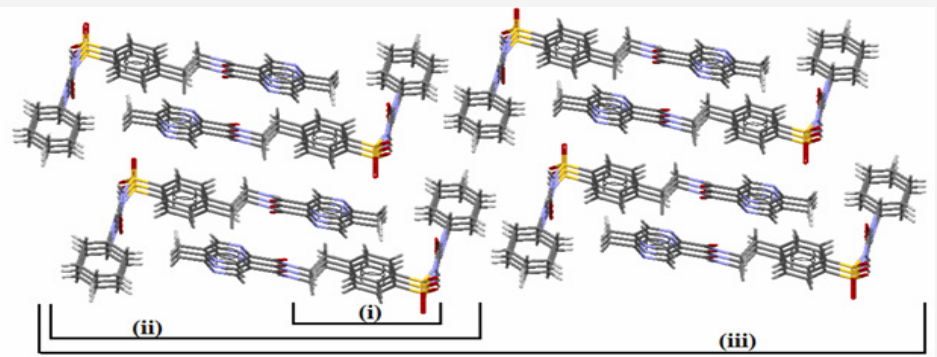

Figure 5 (b)

Figure 5: (a) Homomeric interactions in GPZ (b) arrangements of GPZ molecules in crystal lattice along a* axis; i) hydrogen bonded parallel GPZ molecules in chains, ii) interlocked pair, iii) layers linked via van der Waals forces (CCDC No: 292334 and 1516974, Refcode: SAXFED and SAXFED01) [21-22]. 
The crystal structure of GPZ is first reported by Burley [25] using PXRD and recently corroborated by Samie et al. [26] group using the single crystal x-ray diffraction. GPZ crystallizes in triclinic crystal system with P-1 space group. The hydrogen atom of one $\mathrm{NH}_{\text {sulfonamide (sulfonylurea) }}$ of GPZ interacts with oxygen atom of $-\mathrm{SO}_{\text {2sulfonylurea }}$

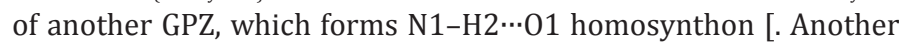
$-\mathrm{NH}_{\text {amide (sulfonylurea) }}$ of GPZ forms bifurcated hydrogen bonding with oxygen atom of $-\mathrm{CO}_{\text {sulfonylurea }}$ and oxygen atom of $-\mathrm{SO}_{2 \text { sulfonylurea }}$ and generates $\mathrm{N} 2-\mathrm{H} 3 \cdots 03$ and $\mathrm{N} 2-\mathrm{H} 3 \cdots 01$ synthons. Besides this, $-\mathrm{NH}_{\text {amide }}$ of GPZ is hydrogen bonded to oxygen of $-\mathrm{CO}_{\text {amide }}$ of another

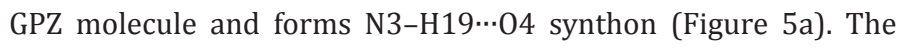
molecules of GPZ are sustained by 1D infinite chained pattern. These chains are packed in pairs, in an interlocking pattern and form layers, which are further linked to other layers via weak van der Waals forces (Figure 5b).

The insertion of the coformers in the crystal lattice of GPZ replaces the GPZ-GPZ interactions with GPZ-coformer interactions, resulting in the generation of cocrystals. GPZ-PA crystallizes in triclinic crystal system with P-1 space group, consisting one molecule of GPZ and one molecule of PA in asymmetric unit cell. The formation of cocrystal disrupts the already present homomeric N$\mathrm{H} \cdots \mathrm{O}$ interactions in GPZ and establishes $\mathrm{N}-\mathrm{H} \cdots \mathrm{N}$ and $\mathrm{O}-\mathrm{H} \cdots \mathrm{O}$ type interactions in the crystal lattice of GPZ-PA. A new heterosynthon (05-H28‥03; [] has formed between the oxygen atom of $\mathrm{CO}_{\text {sulfonylurea }}$ of GPZ and hydrogen atom of $-\mathrm{OH}_{\text {carboxylic }}$ of PA. The -

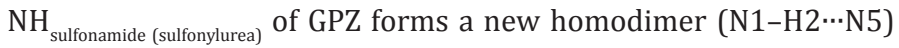
with $\mathrm{N}_{\text {ring }}$ of another GPZ molecule, by breaking the previous N1H2 $\cdots 01$ homosynthon. The GPZ molecules of two asymmetric units are sustained by ring motif. Besides this, the other $-\mathrm{NH}_{\text {amide }}$ (sulfonylurea), which was involved in the homosynthon of GPZ, is now

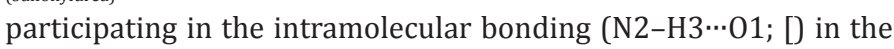
GPZ-PA (Figure 6a). In the crystal lattice of cocrystal, the repetitive entity of two bonded asymmetric units, stabilized via van der Waals forces (can be viewed along c-axis), forms the three-dimensional network of the crystal lattice (Figure 6b).

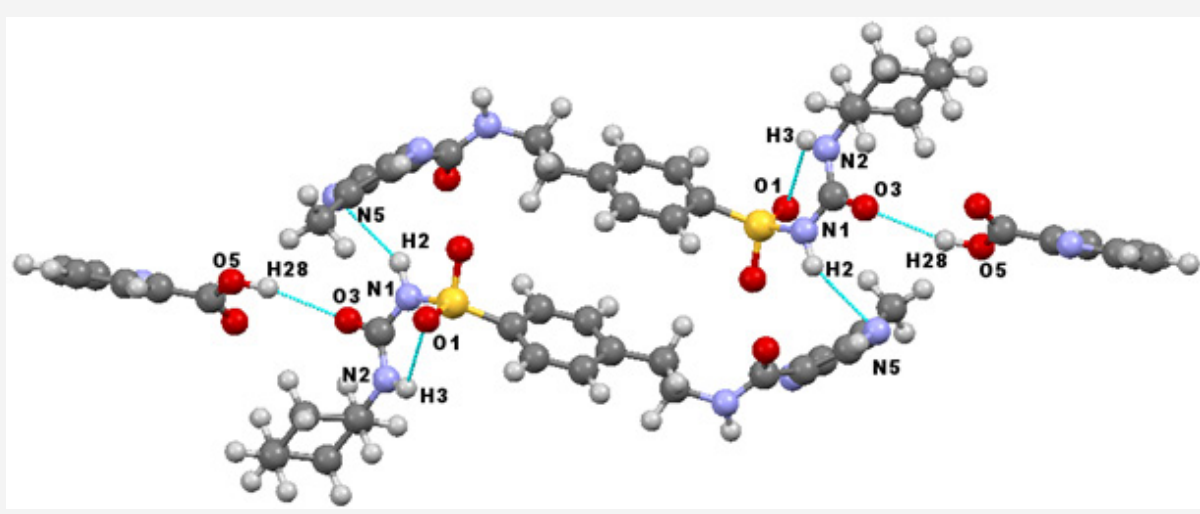

Figure 6(a)

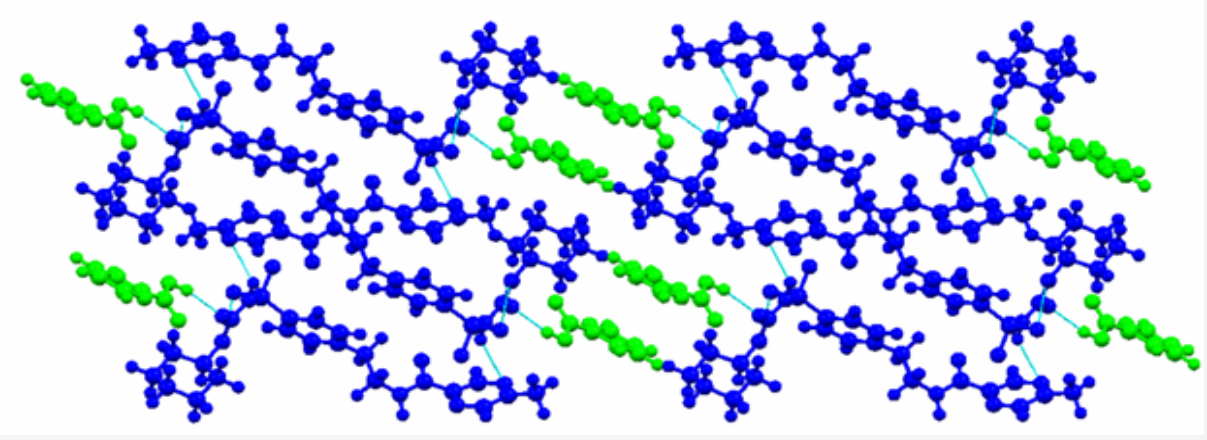

Figure 6(b)

Figure 6: Crystal structure of GPZ-PA: (a) hydrogen bonding in GPZ and PA, (b) 2D view of the arrangement of GPZ and PA molecules along c-axis; GPZ and PA are represented by blue and green color respectively.

GPZ-AA also crystallizes in a triclinic crystal system with P-1 space group. The asymmetric unit encloses one molecule of GPZ and one molecule of AA. In the crystal structure of the cocrystal, -

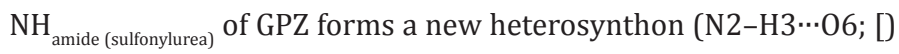
with oxygen of $-\mathrm{CO}_{\text {carboxylic }}$ of $\mathrm{AA}$, which has replaced $\mathrm{N} 2-\mathrm{H} 3 \cdots \mathrm{O} 3$ and N2-H3 ‥01 homosynthon in pure GPZ. Two more heterosynthons are formed between $\mathrm{N}_{\text {ring }}$ of GPZ and hydrogen atoms of both -

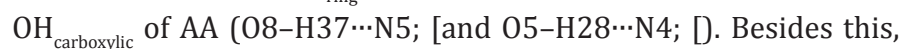
GPZ molecules are attached through a single point homosynthon (N3-H19..03; [) which is formed between hydrogen atom of -
$\mathrm{NH}_{\text {amide }}$ and oxygen atom of $-\mathrm{CO}_{\text {amide }}$ of two different GPZ molecules (Figure 7a) and was absent in the crystal lattice of pure GPZ. The crystal structure reveals that GPZ molecules are present in the folded 'hook' shaped conformations in the crystal lattice and are arranged in such a manner that the hook of two molecules faces each other. This leads to the interactions, forming the infinite chain (along c-axis). The AA molecules seem to be fitted in the cavities formed by aromatic rings of alternate GPZ molecules (Figure $7 \mathrm{~b} \&$ c). 


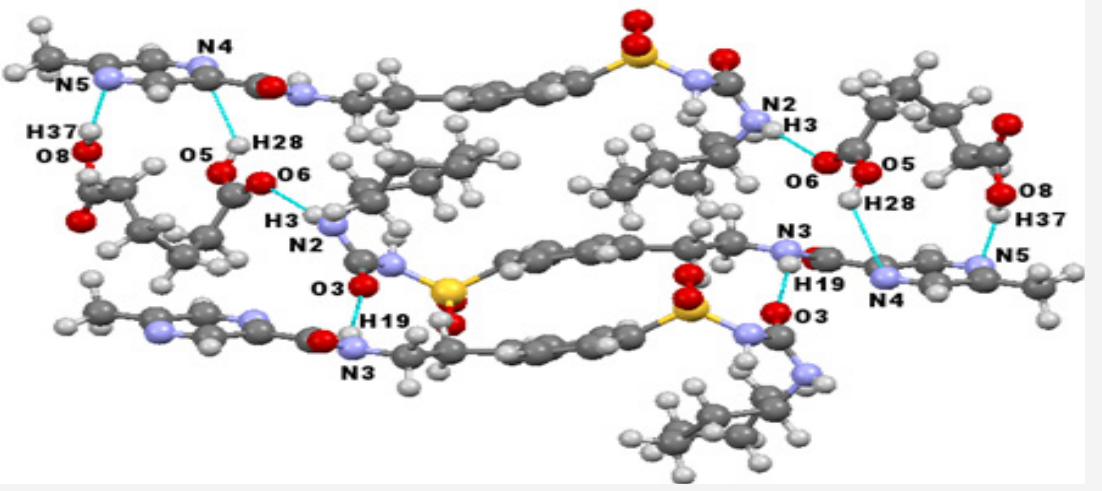

Figure 7(a)

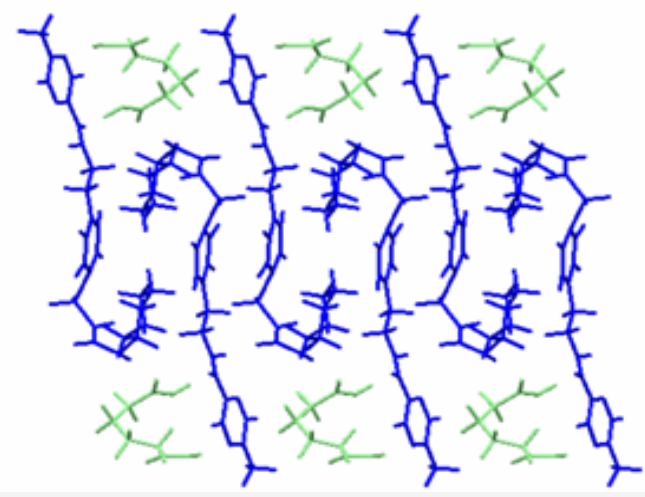

Figure 7(b)

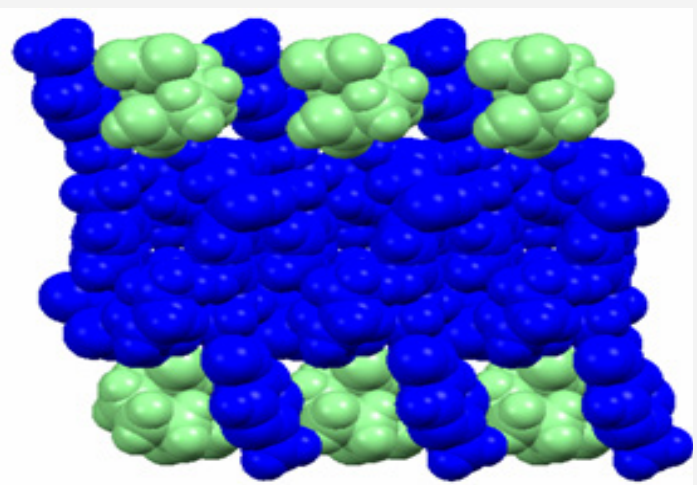

Figure 7(b)

Figure 7: Crystal structure of GPZ-AA: (a) hydrogen bonding between GPZ and AA, (b) 2D packing view of GPZ and AA molecules along c-axis, (c) 3D crystal structure of GPZ-AA in space fill mode; GPZ and AA are represented by blue and green color respectively.

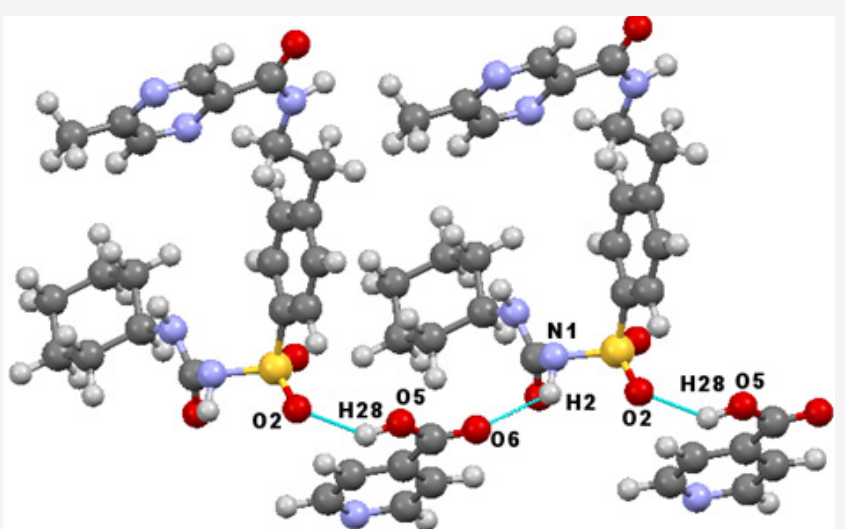

Figure 8 (a)

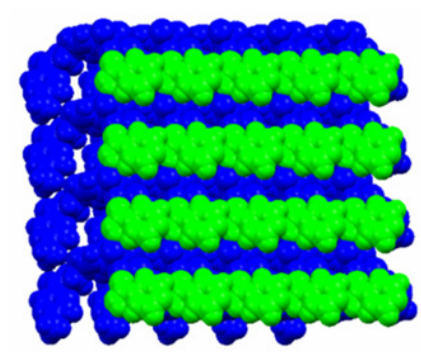

Top view

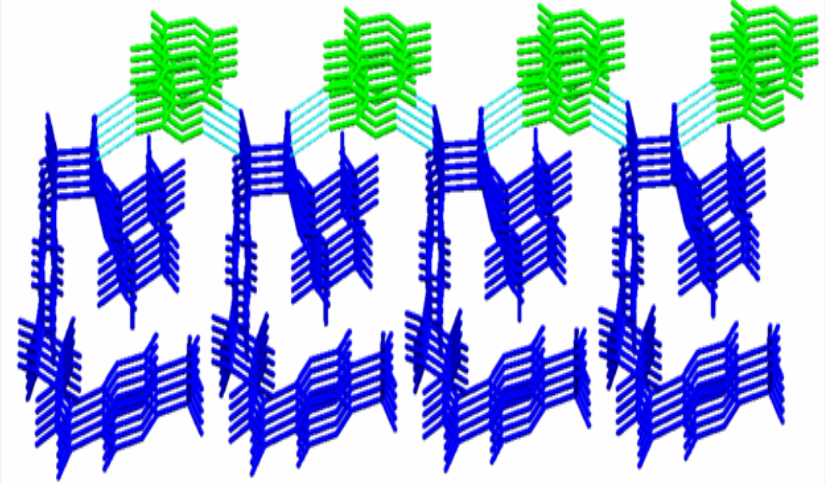

Figure 8 (b)

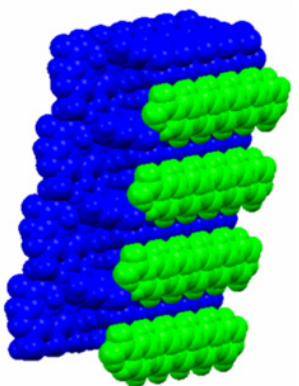

side view

Figure 8 (c)

Figure 8: Crystal structure of GPZ-INA: (a) hydrogen bonding between GPZ and INA, (b) 2D packing view of GPZ and FA molecules along $\mathrm{C}^{*}$-axis, (c) the top and side view of 3D crystal structure of GPZ-INA in space fill mode; GPZ and INA are represented by blue and green color respectively. 
The crystal structure of GPZ-INA reveals that it crystallizes in triclinic system (P1 space group), contains one molecule of GPZ and one molecule of INA in asymmetric unit. Two new heterosynthon

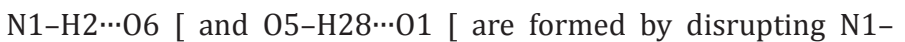

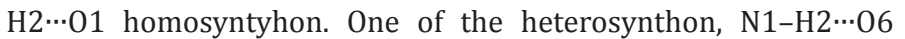
is formed between $-\mathrm{NH}_{s}$ of GPZ and oxygen of $-\mathrm{CO}_{\text {carboxylic }}$ of INA. The other one, $05-\mathrm{H} 28 \cdots 01$ is formed by the hydrogen bonding between oxygen of $-\mathrm{SO}_{\text {2sulfonylurea }}$ of $\mathrm{GPZ}$ and hydrogen of $-\mathrm{OH}_{\text {carboxylic }}$ of INA (Figure 8a). In the crystal lattice, GPZ molecule exists in folded conformation and is connected to another GPZ molecule through a bridge of INA molecule. Both molecules are arranged alternatively and form an infinite chain. These chains are stacked over one another and attached through van der Waals forces (along $\mathrm{c}^{*}$-axis). In the space fill mode of three-dimensional supramolecular networks INA molecules seems to be entangled in the grooves formed by GPZ molecules (Figure 8b \& c).
GPZ-FA belongs to the P-1 space group of triclinic crystal system and contains one molecule of GPZ and one molecule of FA in an asymmetric unit. In the presence of FA, all the preexisting homosynthons in GPZ were replaced by new homo and heterosynthons in the crystal lattice of GPZ-FA. A new one-point heterosynthon is formed between $\mathrm{N}_{\text {ring }}$ of GPZ and hydrogen atom of

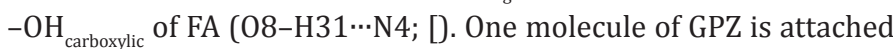
to another GPZ molecule through N3-H19-.03; [ synthon (formed between hydrogen of $-\mathrm{NH}_{\text {amide }}$ and oxygen of $-\mathrm{CO}_{\text {sulfonylurea }}$ ). Besides this, one molecule of FA is connected to another FA molecule via 05-H28*06; [ synthon (formed between hydrogen of $-\mathrm{OH}_{\text {carboxylic }}$ and oxygen of $-\mathrm{CO}_{\text {carboxylic }}$ ) (Figure 9a). In the crystal lattice, the molecules are arranged in an infinite chained wavy pattern along c-axis, in such a way that a pair of GPZ molecule is bridged by a pair of FA molecules (Figure $9 b \& c$ ).

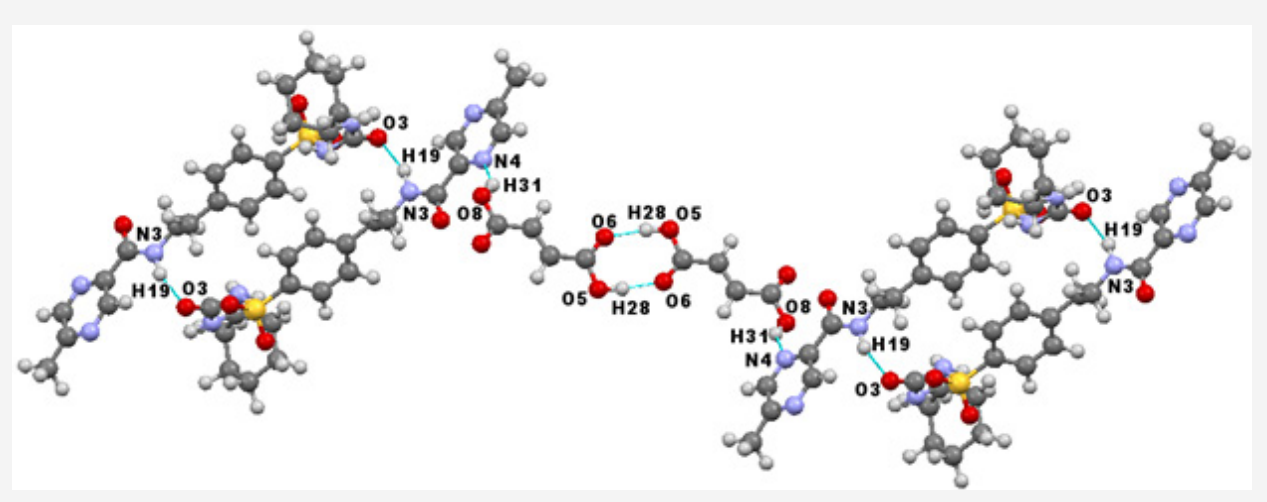

Figure 9 (a)

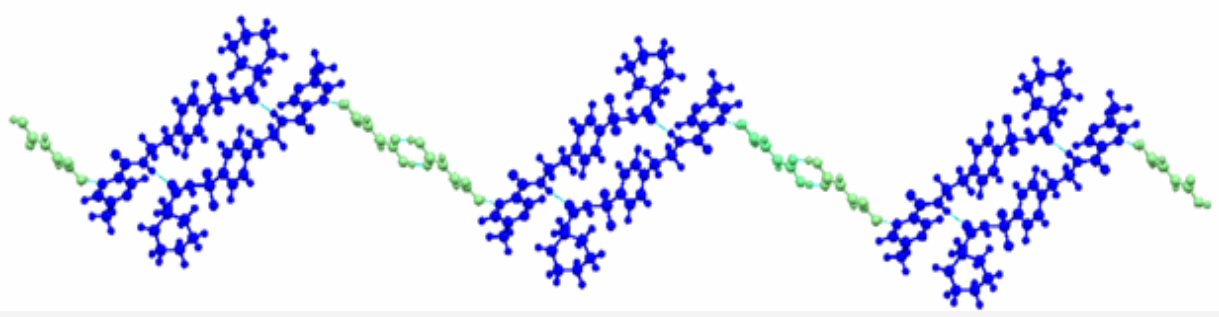

Figure 9(b)

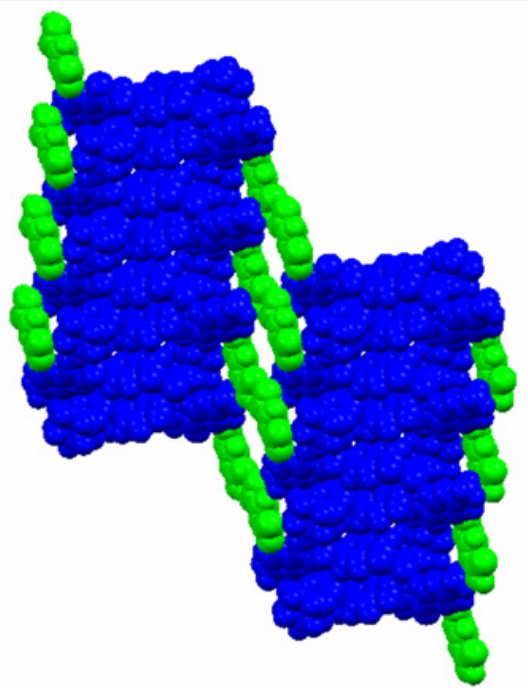

Figure 9(c)

Figure 9: Crystal structure of GPZ-FA: (a) hydrogen bonding between GPZ and FA, (b) 2D packing view of GPZ and FA molecules along c-axis, (c) 3D crystal structure of GPZ-FA in space fill mode along c-axis; GPZ and FA are represented by blue and green color respectively. 
GPZ-SRA was found to exist in monoclinic crystal system with P21 space group and consist of one molecule of GPZ and one molecule of SRA in asymmetric unit cell. The inclusion of SRA in the crystal lattice of GPZ resulted in the breaking of all the preexisting homosynthons and generated new heterosynthons in GPZ-SRA

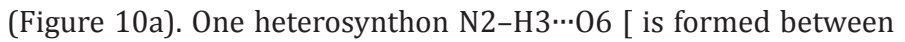
the hydrogen atom of $-\mathrm{NH}_{\text {amide (sulfonylurea) }}$ of GPZ and oxygen atom of $\mathrm{CO}_{\text {carboxylic }}$ of SRA. The oxygen atom of $-\mathrm{SO}_{2 \text { sulfonylurea }}(\mathrm{O} 1)$ of $\mathrm{GPZ}$, which was occupied in the formation of $\mathrm{N} 1-\mathrm{H} 2 \cdots 01$ homosynthon in pure
GPZ, is now hydrogen bonded to hydrogen atom of $-\mathrm{OH}_{\text {carboxylic }}$ of SRA to give a heterosynthon $05-\mathrm{H} 28 \cdots 01$ [. In the crystal lattice, GPZ and SRA molecules are arranged alternatively and sustained by chained synthons. These chains are linked to each other in a parallel manner by van der Waals forces. Upon viewing along b-axis, GPZ molecules seem to be present in dimers, embedded with SRA molecules and these layers constitute the supramolecular network of cocrystal (Figure 10b \& c).

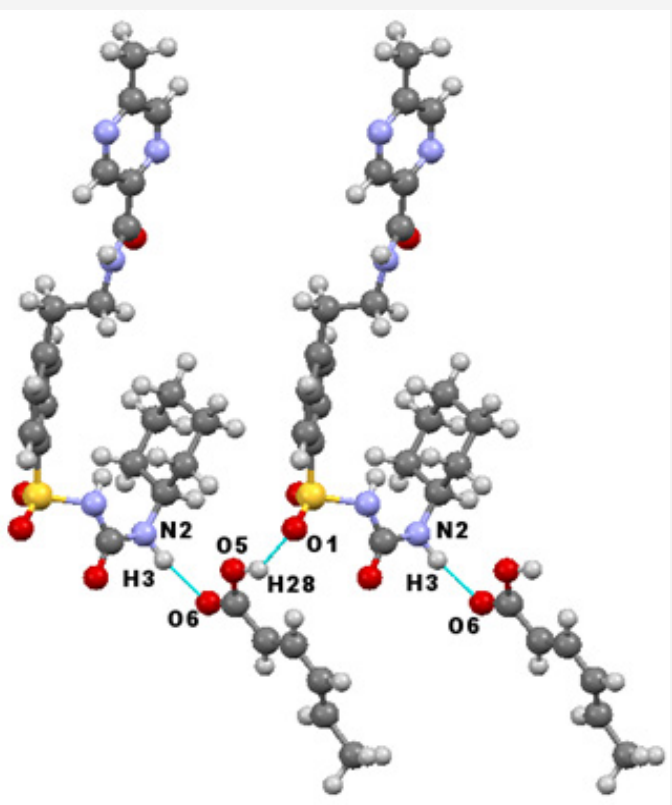

Figure 10 (a)

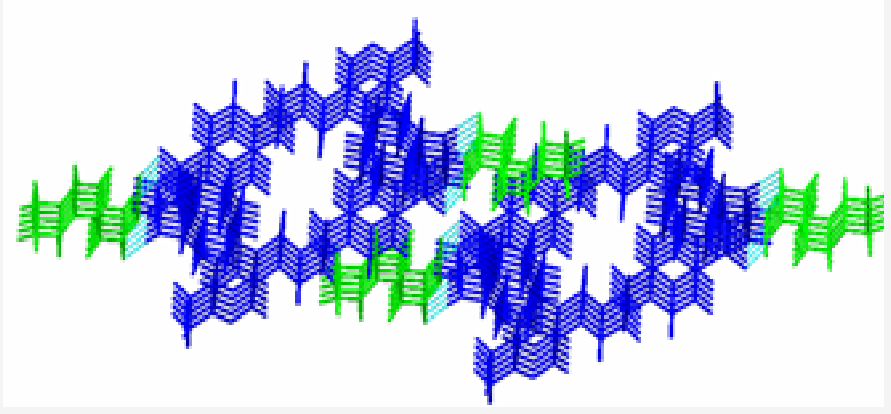

Figure 10 (b)

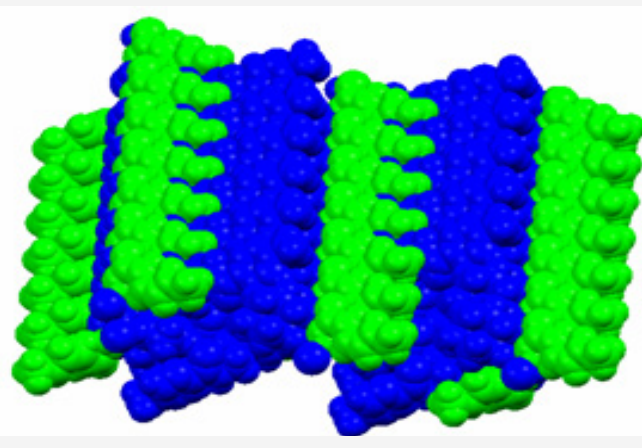

Figure 10 (c)

Figure 10: Crystal structure of GPZ-SRA: (a) the angular displacement of the planes of GPZ and SRA in an asymmetric unit, (b) 2D packing view of GPZ and SRA molecules along b-axis, (c) 3D layers in crystal structure of GPZ-SRA in space fill mode; GPZ and SRA are represented by blue and green color respectively.

Table 1: Crystallographic parameters of cocrystals of GPZ.

\begin{tabular}{|c|c|c|c|c|c|}
\hline Parameters & GPZ-PA & GPZ-AA & GPZ-INA & GPZ-FA & GPZ-SRA \\
\hline Chemical formula & $\mathrm{C}_{21} \mathrm{H}_{27} \mathrm{~N}_{5} \mathrm{O}_{4} \mathrm{~S} ; \mathrm{C}_{6} \mathrm{H}_{5} \mathrm{NO}_{2}$ & $\mathrm{C}_{21} \mathrm{H}_{27} \mathrm{~N}_{5} \mathrm{O}_{4} \mathrm{~S} ; \mathrm{C}_{6} \mathrm{H}_{10} \mathrm{O}_{4}$ & $\mathrm{C}_{21} \mathrm{H}_{27} \mathrm{~N}_{5} \mathrm{O}_{4} \mathrm{~S} ; \mathrm{C}_{6} \mathrm{H}_{5} \mathrm{NO}_{2}$ & $\mathrm{C}_{21} \mathrm{H}_{27} \mathrm{~N}_{5} \mathrm{O}_{4} \mathrm{~S} ; \mathrm{C}_{4} \mathrm{H}_{4} \mathrm{O}_{4}$ & $\mathrm{C}_{21} \mathrm{H}_{27} \mathrm{~N}_{5} \mathrm{O}_{4} \mathrm{~S} ; \mathrm{C}_{6} \mathrm{H}_{8} \mathrm{O}_{2}$ \\
\hline Stoichiometry & $1: 01$ & $1: 01$ & $1: 01$ & $1: 01$ & $1: 01$ \\
\hline Temperature & $\begin{array}{c}\text { Room temperature as } \\
\text { specified } 25^{\circ} \mathrm{C}\end{array}$ & $\begin{array}{c}\text { Room temperature as } \\
\text { specified } 25^{\circ} \mathrm{C}\end{array}$ & $\begin{array}{c}\text { Room temperature as } \\
\text { specified } 25^{\circ} \mathrm{C}\end{array}$ & $\begin{array}{c}\text { Room temperature as } \\
\text { specified } 25^{\circ} \mathrm{C}\end{array}$ & $\begin{array}{c}\text { Room temperature as } \\
\text { specified } 25^{\circ} \mathrm{C}\end{array}$ \\
\hline Crystal system & Triclinic & Triclinic & Triclinic & Triclinic & P-1 \\
\hline Space group & $\mathrm{P}-1$ & $\mathrm{P}-1$ & 24.3602 & 25.6521 & 1 \\
\hline $\mathrm{a}(\AA)$ & 24.0864 & 24.3818 & 9.0222 & 9.6856 & 8.6812 \\
\hline b $(\AA)$ & 8.988 & 9.2343 & & \\
\hline
\end{tabular}




\begin{tabular}{|c|c|c|c|c|c|}
\hline c $(\AA)$ & 6.888 & 5.6605 & 6.7457 & 5.488 & 23.0561 \\
\hline$\alpha(\mathrm{deg})$ & 82.02 & 77.92 & 90.36 & 78.56 & 90 \\
\hline$\beta$ (deg) & 91.78 & 93.59 & 93.58 & 93.4 & 105.07 \\
\hline$\gamma(\mathrm{deg})$ & 96.37 & 96.66 & 95.97 & 96.53 & 90 \\
\hline $\mathrm{Z}$ & 2 & 2 & 2 & 2 & 2 \\
\hline Vol. (Å3) & 1467.42 & 1236.87 & 1471.52 & 1326.8 & 2991.39 \\
\hline $2 \theta$ range & $5^{\circ}-45^{\circ}$ & $5^{\circ}-45^{\circ}$ & $5^{\circ}-45^{\circ}$ & $5^{\circ}-45^{\circ}$ & $5^{\circ}-45^{\circ}$ \\
\hline Rwp & 7.62 & 7.8 & 8.52 & 8.06 & 9.25 \\
\hline CCDC No & 1555857 & 1555858 & 1555859 & 1555860 & 1555863 \\
\hline
\end{tabular}

The crystallographic parameters of the cocrystals are summarized in Table 1 whereas the geometrical parameters are given in Table S2. The rietveld fit profile (comparison of simulated and experimental PXRD pattern) has been given in the supporting information (Figure S3). The obtained Rwp values for all the cocrystals are in the acceptable range (Table 1 ).

\section{SSNMR}

The formation of cocrystals i.e., new crystalline phase is always coupled with change in chemical environment, which arises due to the changes in the interactions between drug and coformers.
The solid state ${ }^{13} \mathrm{C}$ NMR along with crystal structure provides the irrefutable evidence of hydrogen bonding, leading to the cocrystal formation, particularly in cases where single crystals are difficult to isolate.

The solid state ${ }^{13} \mathrm{C}$ NMR spectra of cocrystals of GPZ (Figure 11), in comparison to GPZ and respective coformers also supported the crystal structures, determined from PXRD pattern. The significant changes are observed in the neighboring carbon atoms of nitrogen and oxygen atoms. The note worthy changes is observed in the ${ }^{13} \mathrm{C}$ chemical shift of C2, C3, C4, C14, C15, C16, C17, C18 and C20 of GPZ and C22 of coformers (Table 2).

Table 2: ${ }^{13} \mathrm{C}$ Chemical shift of GPZ and its cocrystals.

\begin{tabular}{|c|c|c|c|c|c|c|}
\hline Carbon Number & GPZ & GPZ-PA & GPZ-AA & GPZ-INA & GPZ-FA & GPZ-SRA \\
\hline C2 & 137 & 136.86 & 136.68 & 136.91 & 136.71 & 137.37 \\
\hline C3 & 159.32 & 158.95 & 159.74 & 158.9 & 159.58 & 159.03 \\
\hline C4 & 51.12 & 51.43 & 51.47 & 51.39 & 51.38 & 51.38 \\
\hline C14 & 42.32 & 42.59 & 42.57 & 42.6 & 42.5 & 42.5 \\
\hline C15 & 166.06 & 165.5 & 165.57 & 165.48 & 165.39 & 165.37 \\
\hline C16 & 142.98 & 143.16 & 143.2 & 142.74 & 143.13 & 143.16 \\
\hline C17 & 144.78 & 145.01 & 144.96 & 144.97 & 144.99 & 144.69 \\
\hline C18 & 151.27 & 151.5 & 151.46 & 151.04 & 151.4 & 151.36 \\
\hline C20 & 143.83 & 144.04 & 144.06 & 143.07 & 143.64 & 143.68 \\
\hline C22 & & 166.18 & 181.66 & 166.34 & 172.07 & 173.59 \\
\hline & & $(166.50$ in PA $)$ & $(182.12$ in AA) & $(166.83$ in INA) & $(172.89$ in FA) & $(174.18$ in SRA) \\
\hline
\end{tabular}

In GPZ-PA, the peak corresponding to C2 (attached to S1 atom) shifted upfield, which supports the involvement of 01 (attached to $\mathrm{S} 1$ atom) in the formation of new intramolecular hydrogen bond, $\mathrm{N} 2-\mathrm{H} 3 \cdots \mathrm{O} 1(\mathrm{r}(\mathrm{H} \cdots \mathrm{A}): 2.712 \AA$ ) by replacing intermolecular $\mathrm{N} 2-$ H3 $\cdots 01$ ( $\mathrm{r}(\mathrm{H} \cdots \mathrm{A}): 2.592 \AA$ ) hydrogen bond. The upfield shifting of $\mathrm{C} 3$ (attached to 03) is indicative of formation of $05-\mathrm{H} 28 \cdots 03$

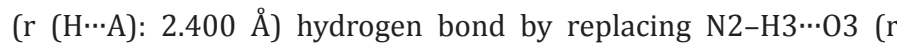
$(\mathrm{H} \cdots \mathrm{A}): 2.196 \AA$ A $)$ hydrogen bond. Moreover, the downfield shift was observed in C4 (attached to N2), which may be due to the no longer availability of $\mathrm{H} 3$ as bifurcated hydrogen donor. Similarily, the downfield shifting of C14 (attached to N3) and upfield shifting

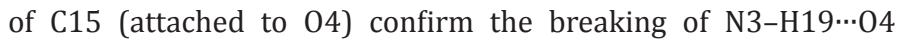
homosynthon. Besides this, the downfield shifting of both $\mathrm{C} 18$ and

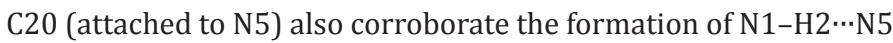
heterosynthon.

The upfield and downfield shifting of C2 (attached to S1) and C3 (attached to 03) respectively, in GPZ-AA, confirm the breaking of N1-H2 $\cdots 01$ synthon. The downfield shifting of C14 (attached to
N3) along with upfield shifting of C15 (attached to 04) substantiate

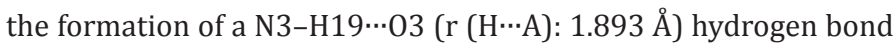

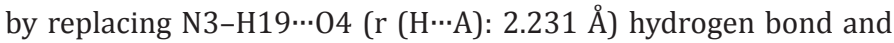
non availability of 04 as an acceptor. The downfield shifting of C4 (attached to N2) may be due to the disappearance of bifurcated hydrogen bonding by $\mathrm{H} 3$ in the cocrystal and formation of N2-

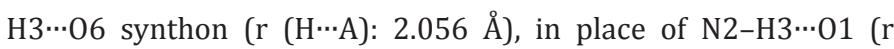
$(\mathrm{H} \cdots \mathrm{A}): 2.592 \AA)$ synthon. Besides this, the downfield shifting of C16, C17, C18, C20 (attached to N4 and N5) and upfield shifting of C22 and C27 (attached to 05, 06, 07 and 08 of AA) shows the presence of $08-\mathrm{H} 37 \cdots \mathrm{N} 5$ and $05-\mathrm{H} 28 \cdots \mathrm{N} 4$ heterosynthon along with the non-availability of 07 as an acceptor.

In GPZ-INA, the upfield shifting of C2 (attached to S1), C3 (attached to 03) and downfield shifting of $\mathrm{C} 4$ (attached to N2) gives an idea of breaking of $\mathrm{N} 1-\mathrm{H} 2 \cdots 01 / \mathrm{N} 2-\mathrm{H} 3 \cdots \mathrm{O} 1$ and $\mathrm{N} 2-$ H3‥03 hydrogen bonds. The marginal shifting of $\mathrm{C} 2$ also indicates the simultaneous involvement of 01 in the formation of another hydrogen bond i.e., 05-H28*06. The downfield shifting of C14 
(attached to N3) along with upfield shifting of C15 (attached to 04),

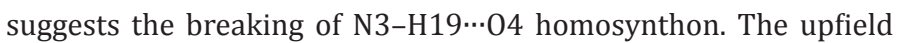
shifting of C22 (attached to 05 and 06) indicates the perturbation in the already present hydrogen bonded network in cofomer and supports the formation of $\mathrm{N} 1-\mathrm{H} 2 \cdots 06$ heterosynthon.

Cocrystal GPZ-FA shows the upfield shifting of C2 (attached to S1) and downfield shifting of C3 (attached to 03) and C4 (attached

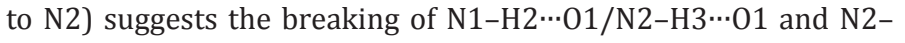

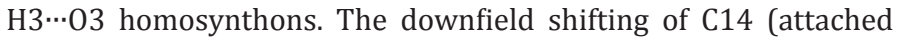

to N3) and upfield shifting of C15 (attached to 04) indicates the

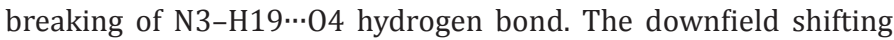
of C3 (attached to 03) and C14 (attached to N3) also confirm the participation of 03 and N3-H19 in the formation of another

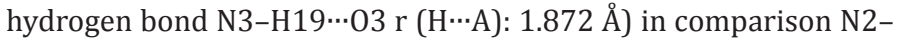
H3 $\cdots 03$ r ( $\mathrm{H} \cdots \mathrm{A}): 2.196 \AA$ ) homosynthon. The upfield shifting of C22 and C25 (attached to 05, 06, 07 and 08) and marginal downfield shifing of C16 and C17 (attached to N4) confirm the presence of 08-H31N 4 synthon.

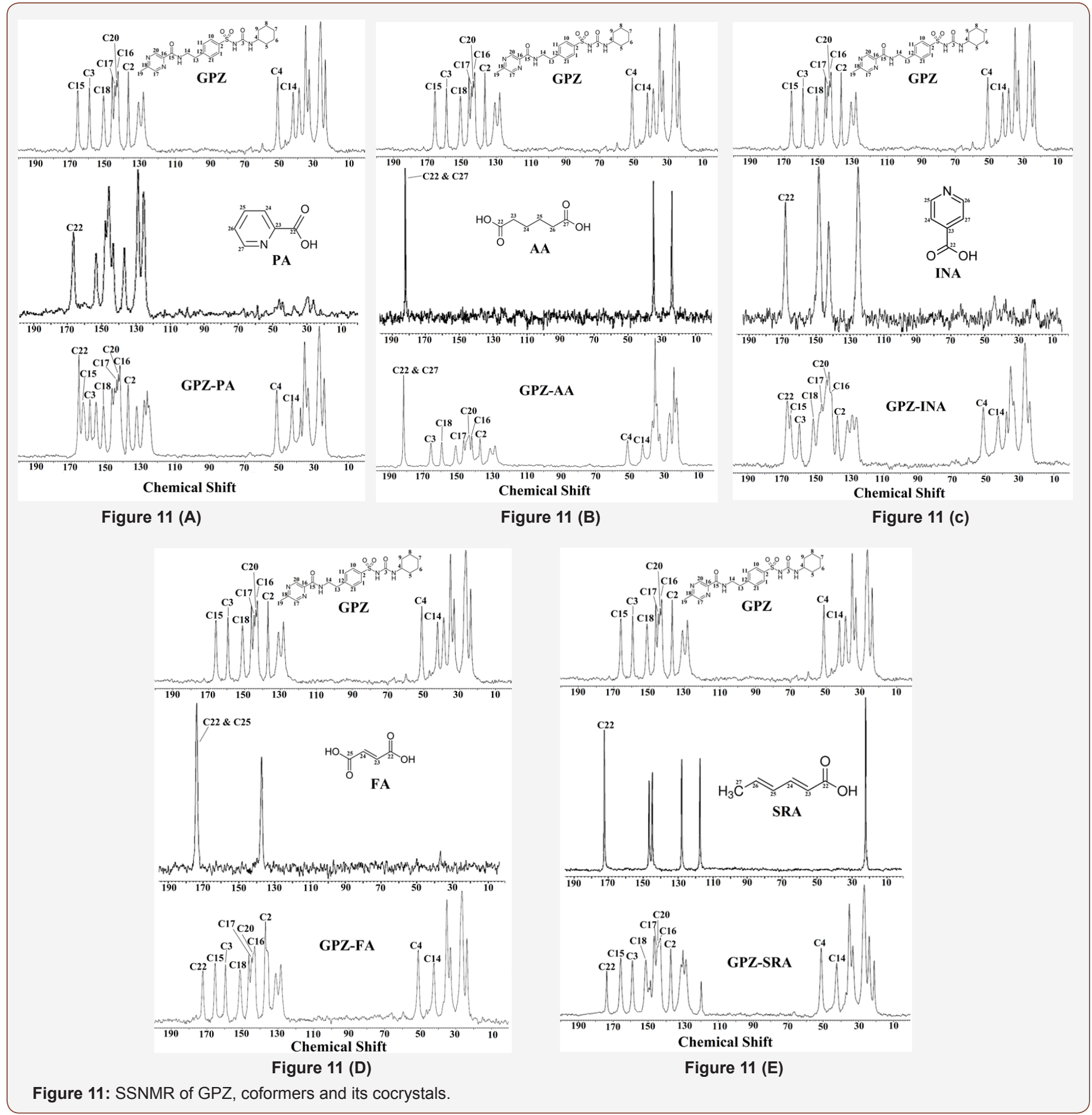

The downfield shifting of C2 (attached to S1) and C4 (attached to N2) is observed in GPZ-SRA, which points towards the breaking of $\mathrm{N} 1-\mathrm{H} 2 \cdots \mathrm{O} 1$ and $\mathrm{N} 2-\mathrm{H} 3 \cdots 01$ homosynthons. Besides this, it

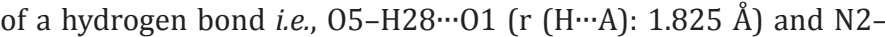

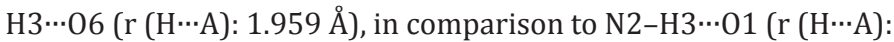
$2.592 \AA$ ) hydrogen bond. The formation of both heterosynthons is also confirms the participation of 01 and N2-H3 in the formation corroborated by upfield shifting of C22 (attached to 05 and 06). 
Besides this, the upfield shifting of C3 (attached to 03), C14 (attached to N2) and downfield shift of $\mathrm{C} 15$ (attached to 04) suggests the

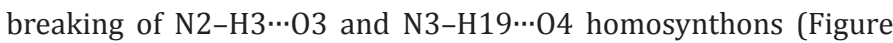
11).

\section{Equilibrium solubility and intrinsic dissolution rate (IDR)}

The determination of equilibrium solubility and IDR of an API in the cocrystals is essential; especially in case of BCS class II drugs where bioavailability is limited by dissolution. The solubility of the coformer and the packing of API and coformer in the crystal lattice are the main contributors in the customization of the same. The cocrystals of GPZ exhibit high solubility and IDR in comparison to GPZ (Table 3). The order of their solubility (Figure 12) and IDR is GPZ-PA ( 6 fold) $>$ GPZ-AA (4.5 fold) > GPZ-INA (3.7 fold) > GPZ-FA (2.3 fold) > GPZ-SRA (1.7 fold). The reason for the best equilibrium solubility and IDR of GPZ-PA may be the highest solubility of PA among all the conformers, used in the present study. GPZ-AA, the second in the solubility order can be explained on the same grounds. If the solubility of the coformers is the sole criterion for deciding the solubility of cocrystals, then GPZ-FA should be more soluble than GPZ-INA, but the observed equililibrium solubility and IDR is just reverse. This can be explained on the basis of crystal structure. It has been detailed in previous section that the strong homomeric interactions is prevailing in GPZ-FA, whereas GPZ-INA has dominating heteromeric interactions. The presence of GPZ dimer in GPZ-FA, poses the difficulties in the breaking of the crystal lattice. However, the low solubility and IDR of GPZ-SRA is only due to its low solubility (Table 3 \& Figure 12).

Table 3: Equilibrium solubility and IDR of GPZ and its cocrystals ${ }^{\dagger}$.

\begin{tabular}{|c|c|c|}
\hline & Solubility $(\mathrm{mg} / \mathrm{mL}) \pm \mathrm{SD}$ & IDR $\left(\mathrm{mg} / \mathbf{m i n} / \mathbf{c m}^{2}\right) \pm \mathrm{SD}$ \\
\hline GPZ & $0.19 \pm 0.02$ & $0.68 \pm 0.02$ \\
\hline GPZ-PA & $1.15 \pm 0.04$ & $4.10 \pm 0.04$ \\
\hline GPZ-AA & $0.85 \pm 0.03$ & $3.08 \pm 0.03$ \\
\hline GPZ-INA & $0.70 \pm 0.02$ & $2.71 \pm 0.02$ \\
\hline GPZ-FA & $0.44 \pm 0.02$ & $1.49 \pm 0.03$ \\
\hline GPZ-SRA & $0.32 \pm 0.03$ & $1.18 \pm 0.02$ \\
\hline
\end{tabular}

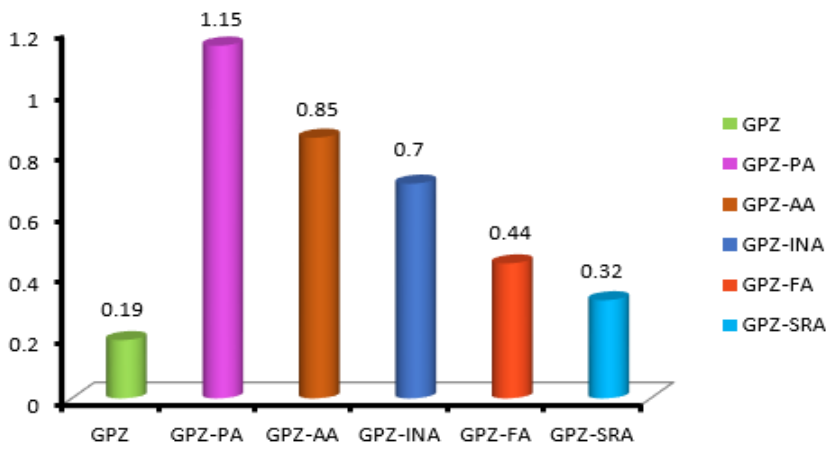

Figure 12: Equilibrium solubility of GPZ and its cocrystals (in mg/ $\mathrm{ml}$ )

\section{In Vivo studies}

The enhancement in the solubility and IDR of an API tailors the pharmacodynamic activity and pharmacokinetic parameters.
All the cocrystals are found to be more effective in reducing blood glucose level (Figure 13) as compared to GPZ, in diabetic rats. The order of reducing blood glucose quantitatively is GPZ-PA> GPZAA> GPZ-INA> GPZ-FA> GPZ-SRA. C $_{\text {max }}$ and AUC (Table 4) for all the cocrystals have been increased in comparison to GPZ, which indicates the enhanced availability of GPZ. Besides this, $\mathrm{T}_{\max }$ is not altered significantly. All these findings point towards the increase in the extent of absorption of GPZ, without affecting the rate of absorption. All the cocrystals have better profile than GPZ and this may provide the viable alternatives for improving the regimen of diabetes by reducing the dose of GPZ (Figure 13 \& Table 4).

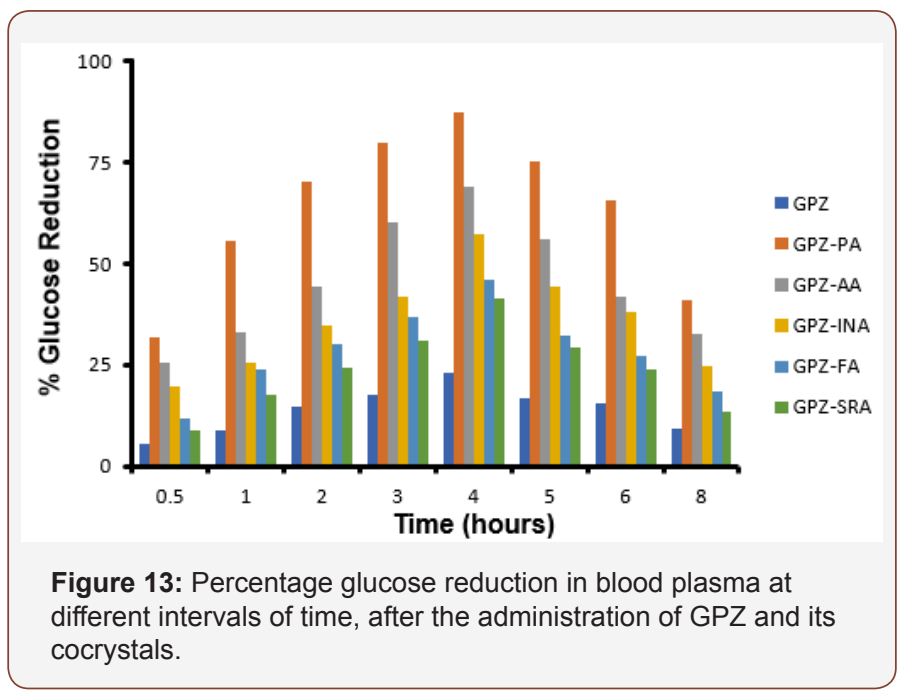

Table 4: Percentage glucose reduction in blood plasma at different intervals of time, after the administration of GPZ and its cocrystals.

\begin{tabular}{|c|c|c|c|}
\hline & $\begin{array}{c}\text { AUC0-t } \pm \text { SEM } \\
(\mathbf{n g} / \mathbf{m l} * \mathbf{m i n})\end{array}$ & $\begin{array}{c}\text { Cmax } \pm \text { SEM } \\
(\mathbf{n g} / \mathbf{m l})\end{array}$ & Tmax(hours) \\
\hline GPZ & $37.14 \pm 4.2$ & $8.23 \pm 2.1$ & 4 \\
\hline GPZ-PA & $221.16 \pm 3.6$ & $47.02 \pm 1.8$ & 4 \\
\hline GPZ-AA & $165.24 \pm 4.3$ & $36.42 \pm 3.2$ & 4 \\
\hline GPZ-INA & $142.03 \pm 2.5$ & $30.36 \pm 2.4$ & 4 \\
\hline GPZ-FA & $99.54 \pm 4.5$ & $18.70 \pm 2.9$ & 4 \\
\hline GPZ-SRA & $62.41 \pm 2.6$ & $14.01 \pm 3.1$ & 4 \\
\hline
\end{tabular}

\section{Conclusion}

Among all the methods of improving the solubility of APIs, cocrystals are the viable means of modifying the physicochemical properties of preexisting APIs. In the present manuscript, GPZ, a BCS class II drug, was targeted for cocrystallization with the aim of improving its solubility and clinical performance. The cocrystals of GPZ were synthesized using the solvent drop grinding method which is a sustainable green approach. The existence of new crystalline phase upon grinding was identified by DSC and PXRD whereas FTIR spectral analysis imparted the information regarding hydrogen bonds among the complementary functional groups present on GPZ and coformers. The stored information in the PXRD patterns, regarding the positions of atoms in the crystal lattice were used to determine the crystal structures of the cocrystals. The supramolecular network of the cocrystals was further supported by changes in the chemical shifts shown by SSNMR. All the cocrystals exhibited high solubility, IDR and improved pharmacodynamic and 
pharmacokinetic parameters as compared to GPZ. For the given data set, it has been found that solubility of the coformers and the crystal structure of the cocrystals are the main determinant of the solubility and IDR of cocrystals. The study presented herein directs the green and economical route of improving the profile of GPZ, which can potentially be developed into the formulation.

\section{Supporting Information}

DSC thermograms of physical mixture of GPZ and coformers, FTIR spectra, Rietveld plots, geometrical paramaters of crystal structure of cocrystals

\section{Conflict of Interest}

The authors declare no competing financial interest.

\section{Acknowledgement}

The authors are greatly thankful to the University Grants Commission (UGC), New Delhi (F.4-1/2006(BSR)/5-94/2007 dated 03-05-2013) and Council of Scientific \& Industrial Research (CSIR), New Delhi (02(0039)/11/EMR-II) for the financial assistance.

\section{References}

1. Bowmaker GA (2013) Solvent-assisted mechanochemistry. Chem Commun 49(4): 334-348.

2. Shearehouse WC, Waddell DC, Mack J (2009) Curr Opin Drug Discovery Dev 12, 772-783.

3. Mokhtari J, Naimi-Jamal MR, Hamzeali H, Dekamin MG, Kaupp G (2009) ChemSusChem 2(3): 248-254.

4. Strobridge FC, Judaš N, Friščić T (2010) A stepwise mechanism and the role of water in the liquid-assisted grinding synthesis of metal-organic materials. Cryst Eng Comm 12(8): 2409-2418.

5. Braga D, Giaffreda SL, Grepioni F, Pettersen A, Maini L, et al. (2006) Mechanochemical preparation of molecular and supramolecular organometallic materials and coordination networks. Dalton Trans 10 1249-1263.

6. Braga D, Grepioni F (2004) Reactions between or within molecular crystals. Angew Chem Int Ed Engl 43(31): 4002-4011.

7. Pichon A, James SL (2008) An array-based study of reactivity under solvent-free mechanochemical conditions-insights and trends. CrystEngComm 10(12): 1839-1847.

8. Schultheiss N, Lorimer K, Wolfe S (2010) Attempted construction of minoxidil: carboxylic acid cocrystals; 7 salts and 1 cocrystal resulted. Desper J CrystEngComm 12(3): 742-749.

9. Friščić T, Trask AV, Jones W, Motherwell WD (2006) Angew Chem 118: 7708-7712.

10. Friscic T, Jones W (2009) Recent Advances in Understanding the Mechanism of Cocrystal Formation via Grinding. Cryst Growth Des 9(3): 1621-1637.
11. Nguyen KL, Friščić T, Day GM, Gladden LF, Jones W (2007) Terahertz time-domain spectroscopy and the quantitative monitoring of mechanochemical cocrystal formation. Nat mat 6(3): 206-209.

12. Karki S, Friščić T, Jones W (2009) Control and interconversion of cocrystal stoichiometry in grinding: stepwise mechanism for the formation of a hydrogen-bonded cocrystal. Cryst Eng Comm 11(3): 470-481.

13. Bowmaker GA, Chaichit N, Pakawatchai C, Skelton BW, White AH (2008) Solvent-assisted mechanochemical synthesis of metal complexes. Dalton Trans 22: 2926-2928.

14. James SL, Adams CJ, Bolm C, Braga D, Collier P, et al. (2012) Mechanochemistry: opportunities for new and cleaner synthesis. Chem Soc Rev 41(1): 413-447.

15. Patel VP, Patel NM (2009) Evaluation of Some Methods for Preparing Glipizide- $\beta$ Cyclodextrin

Inclusion Complexes. Iranian Journal of Pharmaceutical Sciences 5(4): 191198.

16. Patel D, Chaudhary P, Mohan S, Khatri H (2012) e-Journal of Science \& Technology $7(4)$.

17. Shukla M, Rathore P, Jain A, Nayak S (2010) Enhanced solubility study of glipizide using different solubilization Techniques. Int J Pharm Pharm Sci 2: 46-48.

18. Thakral S, Madan AK (2008) Urea co-inclusion compounds of glipizide for the improvement of dissolution profile. J of Incl Pheno and Macro Chem 60: 203-209.

19. Higuchi T, Connors KA (1965) Solid Dispersion Incorporated Microcapsules: Predictive Tools for Improve the Half Life and Dissolution Rate of Pioglitazone Hydrochloride. Adv Anal Chem Instrum 4: 117-212.

20.Zafar M, Naqvi SN, Ahmed M, Kaimkhani ZA (2009) Altered Kidney Morphology and Enzymes

in Streptozotocin Induced Diabetic Rats. Int J Morphol 27(3): 783-790.

21. Babu RP, Vanishree S, Sujatha S, Kishore KK (2012) Int J of Pharm and Pharm Sci 4: 133-138.

22. Trinder PJ (1969) Determination of blood glucose using an oxidaseperoxidase system with a non-carcinogenic chromogen. J Clin Pathol 22 158-161.

23. Zhang Y, Huo M, Zhou J, Xie S (2010) PKSolver: An add-in program for pharmacokinetic and pharmacodynamic data analysis in Microsoft Excel. Comp Methods and Prog in Biomed 99(3): 306-314.

24. Newman AW, Byrn SR (2003) Solid-state analysis of the active pharmaceutical ingredient in drug products. Drug Discov Today 8(19): 898-905.

25. Burley JC (2005) Act Crystall Sec B: Struc Sci 61: 710-716.

26. Samie A, Desiraju GR, Banik M (2017) Salts and Cocrystals of the Antidiabetic Drugs Gliclazide, Tolbutamide, and Glipizide: Solubility Enhancements through Drug-Coformer Interactions. Cryst Growth Des 17: 2406-2417. 